\title{
Antiskin Inflammatory Activity of Black Ginger (Kaempferia parviflora) through Antioxidative Activity
}

\author{
Myung-hee Lee, ${ }^{1}$ Ah-Ram Han, ${ }^{1}$ Mi Jang ${ }^{1},{ }^{1}$ Hyo-Kyoung Choi, ${ }^{1}$ Sung-Young Lee, ${ }^{2}$ \\ Kyung-Tack Kim, ${ }^{1}$ and Tae-Gyu Lim ${ }^{1}{ }^{1}$
}

${ }^{1}$ Korea Food Research Institute, Wanju-gun, 55365 Jeollabuk-do, Republic of Korea

${ }^{2}$ The Hormel Institute, University of Minnesota, 801 16th Ave NE, Austin, MN 55912, USA

Correspondence should be addressed to Tae-Gyu Lim; tglim83@kfri.re.kr

Received 18 December 2017; Revised 16 January 2018; Accepted 5 February 2018; Published 3 April 2018

Academic Editor: Kota V. Ramana

Copyright ( 2018 Myung-hee Lee et al. This is an open access article distributed under the Creative Commons Attribution License, which permits unrestricted use, distribution, and reproduction in any medium, provided the original work is properly cited.

\begin{abstract}
Kaempferia parviflora (Krachaidum (KD)) is a traditional herbal medicine and has properties that are beneficial for human health. In the current study, we sought to investigate the anti-inflammatory properties of KD extract (KPE). In mouse skin tissue, UV light representing solar wavelengths (sUV) increased COX-2 expression, while treatment with KPE reduced this effect. The anti-inflammatory activity of KPE was confirmed in in vitro models. MAPK signaling pathways were activated by sUV irradiation, and this was also repressed in the presence of KPE treatment. It is assumed that the anti-inflammatory activity of KPE is caused by the antioxidative effect. Furthermore, we confirmed the critical role of oxidative stress in sUV-induced COX2 expression. We analyzed the polyphenol composition of KPE. Of the polyphenols identified, gallic acid, apigenin, and tangeretin were identified as the major polyphenols (at $9.31 \pm 1.27,2.37 \pm 0.14$, and $2.15 \pm 0.19 \mu \mathrm{g} / \mathrm{mg}$ dry weight, resp.). Collectively, these findings show that in the presence of sUV irradiation, KD has anti-inflammatory properties and antioxidative effects in the skin.
\end{abstract}

\section{Introduction}

Kaempferia parviflora (Krachaidum (KD)), commonly known as Thai ginseng, is a member of the Zingiberaceae family [1]. It has long been used as a popular ingredient in health tonics in Thailand, where it is locally known as Kra-chai-dam. It has been reported that the phytochemical components of KD include flavones, flavonol glycosides, phenolic glycosides, and $\beta$-sitosteryl myristate [2]. Various studies have also reported that KD exhibits anti-inflammatory [3], antiallergenic [4], antimycobacterial, antigastric ulcer [5], and antimutagenic activities [6].

Of the various environmental factors that affect the skin, UV light (the major natural source being sunlight) is the most important. Solar UV induces photochemical reactions, which are known to be a major cause of inflammatory skin lesions and skin cancer [7]. The wavelengths within UV are divided into three bands: UVA (320-400 nm), UVB (280$320 \mathrm{~nm})$, and UVC (200-280 nm) [8]. The UV components of sunlight that reach the Earth's surface (daylight UV) are UVA and UVB (290-400 nm). It has been reported that after UV light irradiation, high concentrations of reactive oxygen species can be generated, which then induces cell membrane damage and skin inflammation [9]. As skin cancer has emerged as a major subject of research efforts, numerous studies on the protection of skin from the harmful effects of sunlight have been conducted [9-11].

$\mathrm{PGE}_{2}$ is a widely known prostaglandin (PG) that is known to play an important role as a mediator of acute inflammation and as a regulator of immune response $[12,13]$. Prostaglandin synthesis is promoted by the transcription factor cyclooxygenase (COX). COX exists as two isoenzymes including COX-1, which is continuously expressed and is involved in regulating homeostasis-related functions in the body [14], and COX-2, which is expressed in the presence of stimulatory conditions such as inflammation. In addition, the prostaglandin generated by COX-2 is known to be involved in the inflammatory response as well as cell proliferation [15]. 
In the current study, we obtained an extract of $\mathrm{KD}$ and investigated its anti-inflammatory effects using in vitro and in vivo models.

\section{Materials and Methods}

2.1. Chemicals and Reagents. MTS solution was obtained from Promega (Madison, WI, USA). Dulbecco's modified eagle medium (DMEM), fetal bovine serum (FBS), and penicillin/streptomycin were purchased from Thermo Fisher Scientific (San José, CA, USA). Primary antibodies specific for COX-2, phosphorylated MKK4 ( $\operatorname{Ser}^{257} / \mathrm{Thr}^{261}$ ), phosphorylated SAPK/JNK $\left(\mathrm{Thr}^{183} / \mathrm{Tyr}^{185}\right)$, phosphorylated MKK3 $\left(\operatorname{Ser}^{189}\right) / 6\left(\operatorname{Ser}^{207}\right)$, phosphorylated p44/p42 ERK1/2 $\left(\mathrm{Thr}^{202} / \mathrm{Tyr}^{204}\right)$, total MKK3, phosphorylated c-Jun $\left(\mathrm{Ser}^{73}\right)$, phosphorylated MEK (Ser $\left.{ }^{217 / 221}\right)$, phosphorylated p90RSK $\left(\mathrm{Thr}^{573}\right)$, phosphorylated p38 ( $\left.\mathrm{Tyr}^{180 / 182}\right)$, and total p38 were purchased from Cell Signaling Technology (Danvers, MA). Antibodies against total ERK1/2, total MKK4, total JNK, and total MEK were obtained from Santa Cruz Biotechnology (Santa Cruz, CA, USA).

2.2. Extract Condition. Approximately $1 \mathrm{~g}$ of $\mathrm{KD}$ powder sample was obtained from Thanyaporn (Samutprakarn, Thailand). The powder was mixed with $25 \mathrm{~mL}$ of $80 \%(v / v)$ EtOH. The soluble components were then extracted in $80^{\circ} \mathrm{C}$ hot water using a reflux condenser. The extract was filtered through filter paper number 2 (Whatman, Maidstone, England) and vacuum-concentrated and subsequently dissolved in $5 \mathrm{~mL}$ of distilled water and freeze-dried, for use as samples for the analysis of antioxidant test.

2.3. UPLC-MS/MS Analysis. The analyses were performed using an ACQUITY UPLC system (Waters, Miliford, MA, USA) with ACQUITY UPLC BEH C18 columns $(2.1 \mathrm{~mm} \times 100 \mathrm{~mm}, 1.7 \mu \mathrm{m})$. The mass spectrometer was a Waters Xevo TQ triple-quadrupole mass spectrometer equipped with electrospray ionization (ESI) mode. MassLynx 4.1 (Waters) software was used for data processing. The mobile phase included $0.1 \%$ formic acid aqueous solution (solvent $\mathrm{A}$ ) and $0.1 \%$ formic acid in acetonitrile (solvent $\mathrm{B}$ ), and a gradient elution program was performed: $0-10 \mathrm{~min}$, 99-70\% solvent A; $10-12 \mathrm{~min}, 70-5 \%$ solvent A; $12-14 \mathrm{~min}$, 5-99\% solvent A; and 14-20 min, 99\% solvent A. The flow rate was set at $0.65 \mathrm{~mL} / \mathrm{min}$, and the column temperature was kept at $40^{\circ} \mathrm{C}$, with a total run time of $20 \mathrm{~min}$. The autosampler was conditioned at $4^{\circ} \mathrm{C}$, and the injection volume was $5 \mu \mathrm{L}$. The LC-MS/MS system was operated in negative ESI mode and scanned using multiple reaction monitoring (MRM) mode. The voltages for capillary, cone, and collision energy were set at $2.5 \mathrm{kV}, 20 \mathrm{~V}$, and $24 \mathrm{~V}$, respectively. The gas flow for desolvation and cone was 800 and $50 \mathrm{~L} / \mathrm{h}$, respectively. The source temperature and desolvation gas temperature were 150 and $400^{\circ} \mathrm{C}$, respectively.

2.4. Cell Culture. JB6 $\mathrm{P}^{+}$and $\mathrm{HaCaT}$ cells were cultured in DMEM supplemented with $10 \%$ FBS and $0.1 \%$ penicillin/ streptomycin/neomycin at $37^{\circ} \mathrm{C}$ in a humidified atmosphere of $5 \% \mathrm{CO}_{2}$. Stable transfectants were generously provided from the Dr. Zigang Dong laboratory in Hormel Institute.
Luciferase reporter-transfected JB6 $\mathrm{P}^{+}$cells were constructed as described previously [16].

2.5. Solar UV Irradiation Systems. UVA-340 lamps were purchased from Q-Lab Corporation (Cleveland, OH). The UVA-340 lamps provide the best possible simulation of sunlight in the critical short-wavelength region from $365 \mathrm{~nm}$ down to the solar cutoff of $295 \mathrm{~nm}$ with a peak emission of $340 \mathrm{~nm}$. The percentage of UVA and UVB emitted by the UVA-340 lamps was measured by a UV meter at $94.5 \%$ and $5.5 \%$, respectively.

2.6. Cell Viability. The cytotoxicity of the sample was measured using CellTiter96 Aqueous One Solution (Promega, Madison, WI). The cells were cultured to confluence in 96well plates. KPE was then treated to the cells for $24 \mathrm{~h}$. $20 \mu \mathrm{L}$ of MTS solution was then added for $1 \mathrm{~h}$ at $37^{\circ} \mathrm{C}$ in a $5 \%$ $\mathrm{CO}_{2}$ incubator. The absorbance was evaluated at $492 \mathrm{~nm}$.

2.7. Luciferase Assay for the COX-2 Promoter and NF- $\kappa B$ Transcriptional Activity. JB6 $\mathrm{P}^{+}$cells stably transfected with COX -2 , and NF- $\kappa$ B luciferase reporter plasmids were used for the luciferase assay. Cells reached confluence before the serum was replaced with serum-free DMEM for $24 \mathrm{~h}$. KPE was pretreated to the cells for $1 \mathrm{~h}$. Then, $23 \mathrm{~kJ} / \mathrm{cm}^{2}$ of $\mathrm{sUV}$ was irradiated to the cells and the cells were incubated at $37^{\circ} \mathrm{C}$ in a humidified atmosphere of $5 \% \mathrm{CO}_{2}$. After $4 \mathrm{~h}$ of sUV exposure, the cells were disrupted using lysis buffer $(0.1 \mathrm{M}$ potassium phosphate buffer ( $\mathrm{pH} 7.8), 1 \%$ Triton X-100, 1 mM DTT, and 2 mM EDTA), and luciferase activity was measured using a luminometer (Luminoskan Ascent, Thermo Electron, Helsinki, Finland).

2.8. Western Blot. JB6 $\mathrm{P}^{+}$and HaCaT cells were cultured to $100 \%$ confluency, and the serum was removed for $24 \mathrm{~h}$ to eliminate FBS-mediated signaling activation. KPE was then treated to the cells at various concentrations. After $1 \mathrm{~h}$ of incubation with $\mathrm{KPE}$, the cells were exposed to sUV $\left(23 \mathrm{~kJ} / \mathrm{cm}^{2}\right)$, before lysis using $1 \mathrm{x}$ lysis buffer (Cell Signaling Biotechnology, Beverly, MA). Equal concentrations of the protein samples were separated on polyacrylamide gels (Bio-Rad Laboratories, Hercules, CA) and transferred to Immobilon-P membranes (Millipore, Billerica, MA). The membranes were blocked with $5 \%$ fat-free milk for $1 \mathrm{~h}$ and incubated with specific primary antibodies at $4^{\circ} \mathrm{C}$ overnight. The proteins were then hybridized with HRPconjugated secondary antibodies and detected using a chemiluminescence detection kit (GE Healthcare, Pittsburgh, PA).

2.9. PGE2 Assay. KPE was treated to JB6 $\mathrm{P}^{+}$cells for $1 \mathrm{~h}$, before $\operatorname{sUV}\left(23 \mathrm{~kJ} / \mathrm{cm}^{2}\right)$ was irradiated to the cells. After $4 \mathrm{~h}$, the production of PGE2 was measured from the cell culture media using a PGE2 enzyme immunoassay kit (R\&D Systems, Minneapolis, MN, USA). The experiments were performed in triplicate.

2.10. ABTS and DPPH Radical Scavenging Assay. The ABTS radical-scavenging activity of KPE was measured using a slightly modified method from van den Berg 

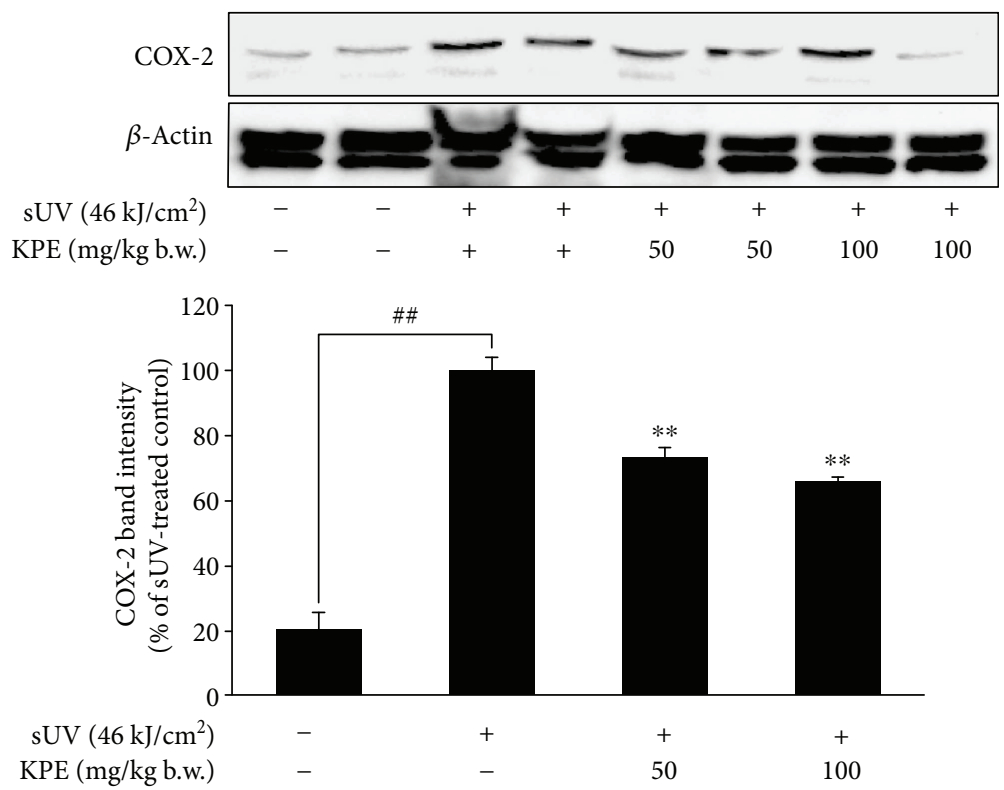

(a)

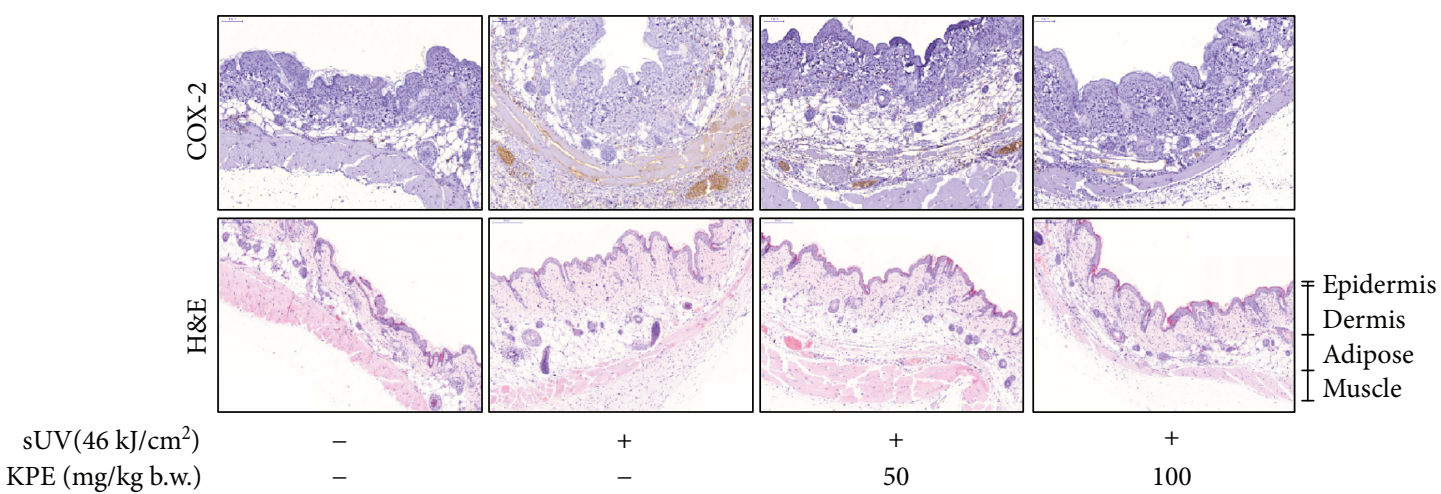

(b)

FIGURE 1: Inhibitory effect of Kaempferia parviflora extract (KPE) on sUV-induced COX-2 expression in mouse skin. (a) Each protein level was detected with the specific primary antibodies. The detailed procedure was described in Materials and Methods. Relative protein expression levels are expressed as the percentage of sUV-treated group intensity, which was set to 100\%. The pound signs (\#\#) and asterisks $(* *)$ indicate significant difference (of $p<0.001$ ) compared to the untreated group and sUV-treated group, respectively. (b) Histopathological analysis of hematoxylin and eosin (H\&E) and COX-2 expression in mouse skin tissue. The detailed procedure is described in Materials and Methods.

et al. [17]. 0.1 M PBS (pH 7.4), 2.5 mM ABTS [2,2' -azinobis-(3-ethylbenzothiazoline-6-sulfonic acid)], and $1.0 \mathrm{mM}$ AAPH [2,2' -azobis(2-mehtylpropionamidine) dihydrochloride] were mixed and maintained for $12 \mathrm{~min}$ in a dark room at $68^{\circ} \mathrm{C}$, then quickly cooled to generate ABTS radical solution. For assessment of the antioxidant capacity, $20 \mu \mathrm{L}$ of the $\mathrm{KD}$ extract was mixed with $980 \mu \mathrm{L}$ of ABTS radical solution and incubated for $10 \mathrm{~min}$ at $37^{\circ} \mathrm{C}$, whereupon absorbance was measured using multimode microplate readers (Infinite ${ }^{\circledR}$ 2000 PRO, Tecan, Switzerland) at $734 \mathrm{~nm}$. DPPH radical scavenging activity was measured as follows; each $0.2 \mathrm{~mL}$ of the $\mathrm{KD}$ extract was added to $3 \mathrm{~mL}$ of ethanol, to which $0.8 \mathrm{~mL}$ of $4 \times 10^{-4} \mathrm{M} \mathrm{DPPH}$ dissolved in ethanol was added. This mixture was vortexed for $10 \mathrm{~s}$ and maintained at room temperature for $10 \mathrm{~min}$, and the absorbance was measured at $517 \mathrm{~nm}$ [18]. The DPPH radical scavenging activity was expressed as a percentage of the absorbance of the group to which no DPPH was added. All experiments were performed at least in triplicate.

2.11. ORAC Assay. The ORAC assay was performed by KOMABIOTECH (Seoul, Korea). The total procedure was followed by the manufacturer's instruction. Briefly, the oxygen radical absorbance capacity was measured by the fluorescence unit. The ORAC activity was represented using Trolox equivalent antioxidant capacity. The experiments were performed in triplicate.

2.12. Animal Study. The animal experimental protocol (2017-0030) was approved, and animals were maintained under specific pathogen-free conditions based on the guidelines established by the Experimental Animal Research 


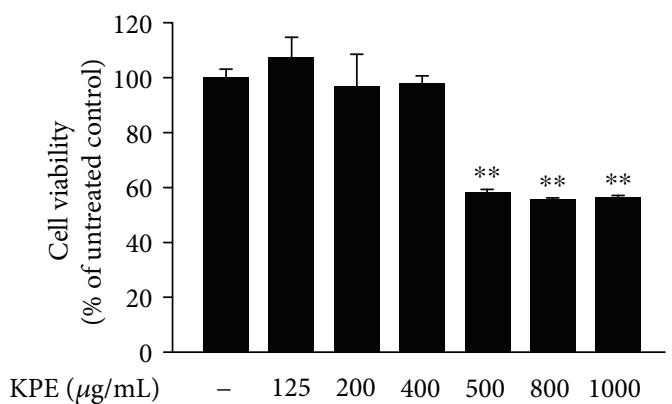

(a)

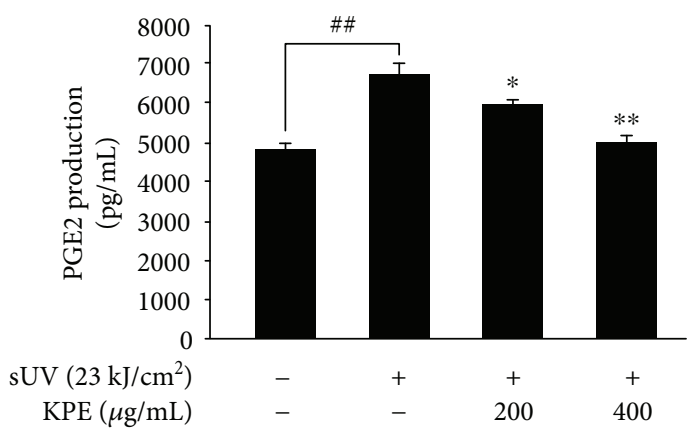

(c)

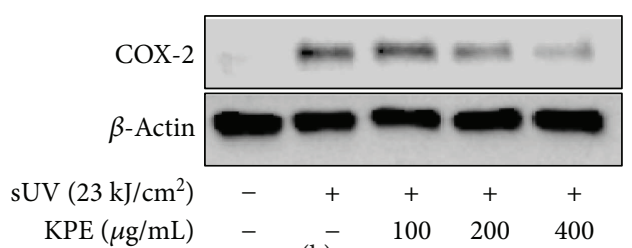

(b)

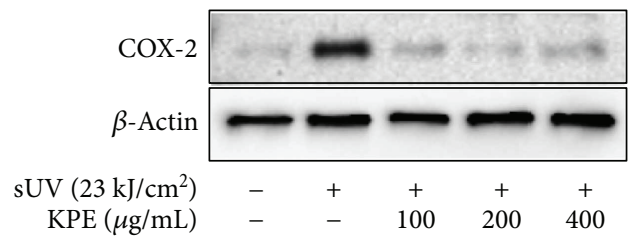

(d)

FIGURE 2: Inhibitory effect of Kaempferia parviflora extract (KPE) on sUV-induced prostaglandin E2 (PGE2) production via COX-2 reduction in JB6 $\mathrm{P}^{+}$and HaCaT cells. (a) The cytotoxicity of KPE was measured using MTS analysis as indicated in Materials and Methods. The COX-2 expression and PGE2 production were determined with Western blot analysis and PGE2 assay in JB6 $\mathrm{P}^{+}(\mathrm{b}, \mathrm{c})$ and $\mathrm{HaCaT}(\mathrm{d})$. KPE was pretreated to the cells for $1 \mathrm{~h}$ before $\mathrm{sUV}\left(23 \mathrm{~kJ} / \mathrm{cm}^{2}\right)$ exposure. After $3 \mathrm{~h}$, the protein was collected. The pound signs $(\# \#)$ and asterisks $(* *)$ indicate significant difference $(p<0.001)$ compared to the untreated group and sUV-treated group, respectively. And the asterisk $(*)$ represents a significant difference $(p<0.01)$ compared to the sUV-treated group.

Laboratory of the Korea Food Research Institute. Female SKH-1 hairless mice ( 5 weeks old, 5 mice in each group) were obtained from Central Lab, Animal Inc. (Seoul, Korea). The animals were housed in climate-controlled quarters $\left(24^{\circ} \mathrm{C}\right.$ at $50 \%$ humidity) with a $12 \mathrm{~h}$ light/dark cycle.

For the experiment, 50 and $100 \mathrm{mg} / \mathrm{kg}$ b.w. of KPE in DMSO were applied to the dorsal skin of mice for $1 \mathrm{~h}$, before the animals were exposed to $46 \mathrm{~kJ} / \mathrm{cm}^{2}$ of $\mathrm{sUV}$. The mice were sacrificed after $4 \mathrm{~h}$, and the dorsal skin sections were collected and the protein was recovered using RIPA buffer.

2.13. Immunohistochemical Staining. Immunohistochemical staining was performed by Abion CRO (Seoul, Korea). Briefly, the skin tissue samples were fixed in $10 \%$ formalin before dehydration using a graded ethanol series. In accordance with general protocols, the tissue was processed for embedding in paraffin wax. To exclude endogenous peroxidase activity, the sections were incubated in $0.3 \% \mathrm{H}_{2} \mathrm{O}_{2}$ for $15 \mathrm{~min}$, and then each primary antibody was treated at a $1: 200$ dilution factor for $1 \mathrm{~h}$. The detection system visualizing anti-mouse antibodies (K4001; DAKO, Glostrup, Denmark) was used according to the manufacturers' instructions. Slides were stained with liquid diaminobenzidine tetrahydrochloride $\left(\mathrm{DAB}^{+}\right)$, a high-sensitivity substrate-chromogen system (K3468; DAKO). The images on the slides were visualized with an Olympus BX40 light microscope.
2.14. Statistical Analysis. All experiments were performed at least three times. Data are expressed as the mean and SD values, and Student's $t$-test was used for single statistical comparisons. A probability cutoff of $p<0.05$ was used as the criterion for statistical significance.

\section{Results}

3.1. Kaempferia parviflora Extract Counteracts UV-Induced Inflammatory Activity in the Skin of Hairless Mice. COX-2 is a representative inflammatory biomarker [15]. Several studies have reported that aberrant increments in COX-2 expression are associated with tumor promotion. Thus, we examined the effect of KPE on sUV-induced COX-2 expression using a hairless mouse model. Briefly, KPE (50 and $100 \mathrm{mg} / \mathrm{kg}$ b.w.) was applied to the dorsal skin of mice for $1 \mathrm{~h}$, before the animals were exposed to $46 \mathrm{~kJ} / \mathrm{cm}^{2}$ of $\mathrm{sUV}$. As seen in Figure 1(a), KPE reduced sUV-induced COX-2 expression and the suppression was confirmed in immunohistological staining (Figure 1(b)).

\subsection{Kaempferia parviflora Extract Inhibits Solar UV-Induced} PGE2 Production via a Reduction in COX-2 Expression in Mouse Epidermal Cells. Production of PGE2 is closely associated with the release of cytokines, such as interleukin- (IL-) $1 \alpha$ and tumor necrosis factor- (TNF-) $\alpha$ [19]. We evaluated the effect of KPE on sUV-induced PGE2 production in JB6 $\mathrm{P}^{+}$cells. The mouse epidermal JB6 $\mathrm{P}^{+}$cell line is a well- 


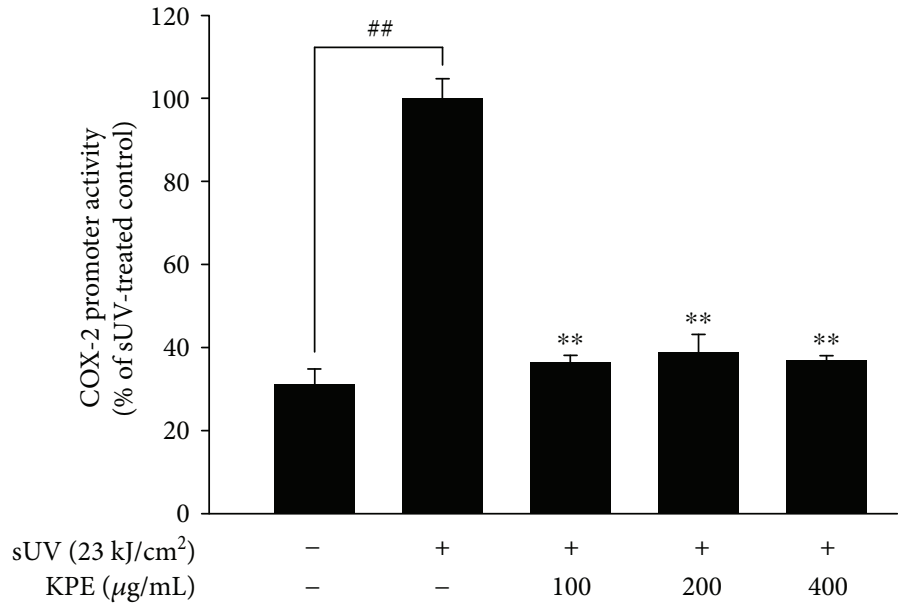

(a)

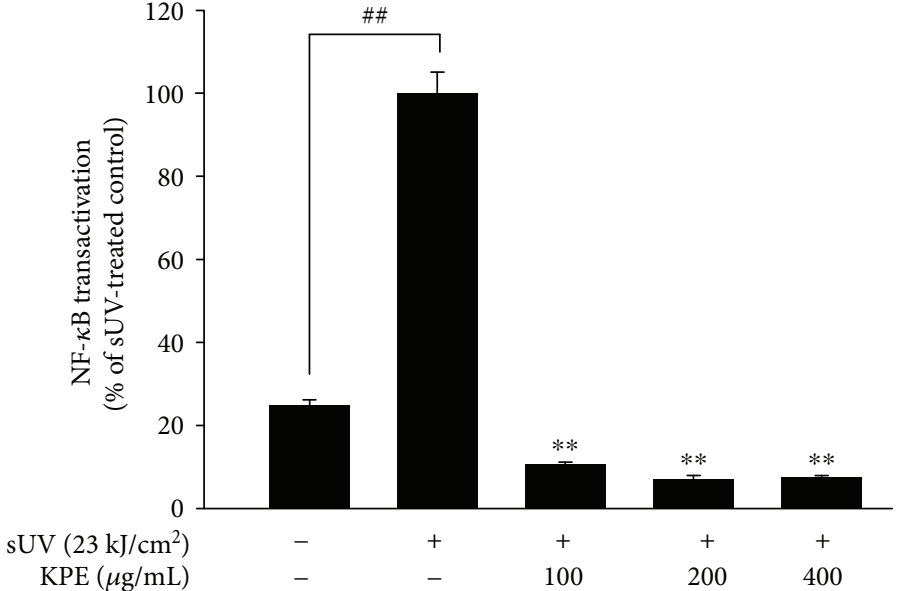

(b)

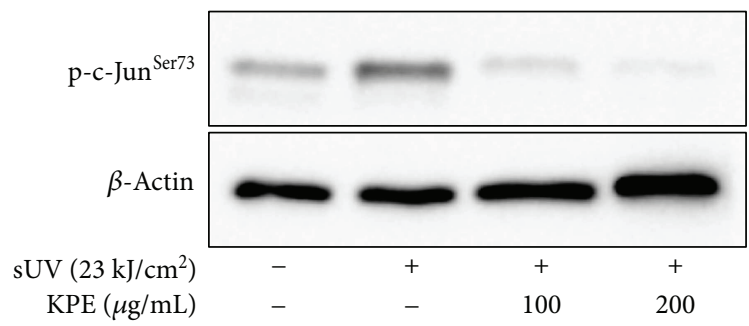

(c)

FIgURE 3: Transcriptional regulation of Kaempferia parviflora extract (KPE) on sUV-induced COX-2 expression. The promoter and transcriptional activity of $\operatorname{cox}-2$ (a) and NF- $\kappa \mathrm{B}$ (b) were downregulated by KPE, respectively. The detailed procedure is indicated in Materials and Methods. Briefly, KPE was pretreated to the cells prior to sUV $\left(23 \mathrm{~kJ} / \mathrm{cm}^{2}\right)$ irradiation. After $3 \mathrm{~h}$ of sUV exposure, each luciferase activity was measured. The pound signs (\#\#) and asterisks $(* *)$ indicate significant difference (of $p<0.001)$ compared to the untreated group and sUV-treated group, respectively. (c) The phosphorylation level of c-Jun was detected using Western blot assay with a specific primary antibody. Data are representative of 3 independent experiments that gave similar results.

established cell line for assessing novel anti-inflammatory agents. Similar with findings from a previous study [19], sUV irradiation statistically enhanced PGE2 production in JB6 $\mathrm{P}^{+}$cells, while pretreatment with KPE attenuated sUVinduced PGE2 production in a dose-dependent manner (Figure 2(c)). The dose range of KPE did not exhibit any cell cytotoxicity up to concentrations of $400 \mu \mathrm{g} / \mathrm{mL}$ (Figure 2(a)). Additionally, sUV-induced COX-2 expression was downregulated by KPE treatment (Figure 2(b)). To obtain more physiologically relevant findings, we assessed the effect of KPE on human keratinocyte $\mathrm{HaCaT}$ cells. The inhibitory effect of KPE on sUV-induced COX-2 expression was also reflected in the HaCaT cells (Figure 2(d)).

3.3. Kaempferia parviflora Extract Modulates sUV-Induced COX-2 Expression via Transcriptional Regulation. It has been reported that mutated DNA-binding sites within NF- $\kappa \mathrm{B}$ and AP-1 can cause a significant attenuation of cox-2 gene expression [20]. AP-1 and NF- $\kappa \mathrm{B}$ are major transcription factors for sUV-induced COX-2 expression [21]. We evaluated the transcriptional activity of NF- $\kappa \mathrm{B}$ as well as $c o x-2$ promoter activity using stably transfected JB6 $\mathrm{P}^{+}$cells. In Figure 3(a), the promoter activity of cox-2 was enhanced after $3 \mathrm{~h}$ of sUV exposure, and treatment with KPE for $1 \mathrm{~h}$ effectively reduced the cox- 2 promoter activity down to control levels. Figure 3(b) shows a similar trend seen in the cox-2 promoter assay. The irradiation with sUV increased NF- $\kappa \mathrm{B}$ transcriptional activity, while KPE treatment suppressed this increment (Figure 3(b)). AP-1 is a complex comprised of Jun family (c-Jun, JunB, and JunD) and Fos family members (c-fos, FosB, Fra-1, and Fra-2) and is generally present as a heterodimer of c-Jun and c-Fos [22]. Thus, we confirmed whether KPE had any effect on c-Jun phosphorylation. As shown in Figure 3(c), the phosphorylation of c-Jun at Ser ${ }^{73}$ was elevated by sUV, and this increment was suppressed by KPE treatment. 


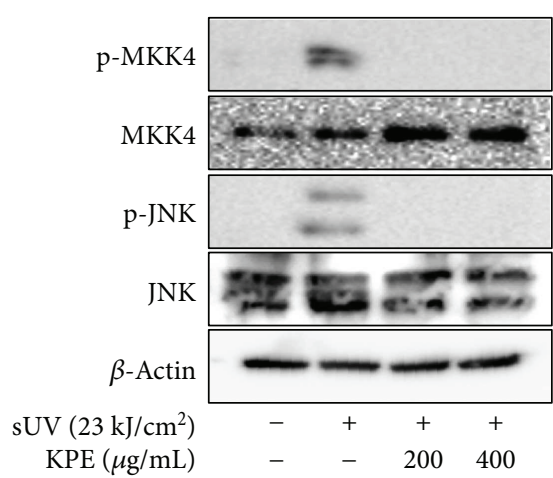

(a)

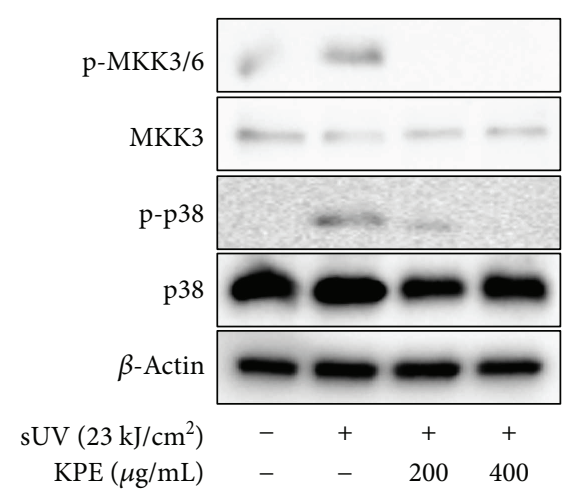

(b)

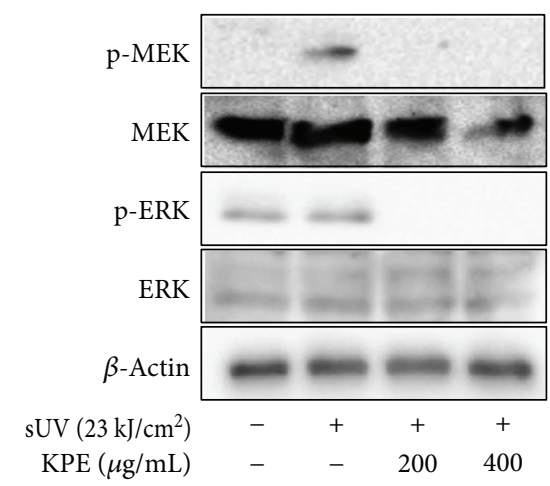

(c)

Figure 4: Inhibitory effect of Kaempferia parviflora extract (KPE) on sUV-induced MKK4-JNK (a), MKK3/6-p38 (b), and MEK-ERK (c) signaling pathway in JB6 $\mathrm{P}^{+}$cells. The detailed method is presented in Materials and Methods. Briefly, KPE was pretreated to the cells for $1 \mathrm{~h}$, and the sUV irradiation step was followed. Each protein level was estimated with primary antibodies. Data are representative of 3 independent experiments that gave similar results.

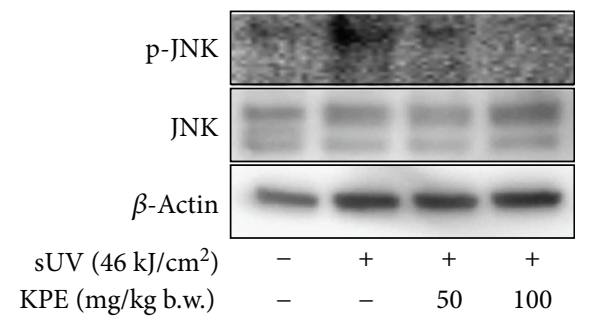

(a)

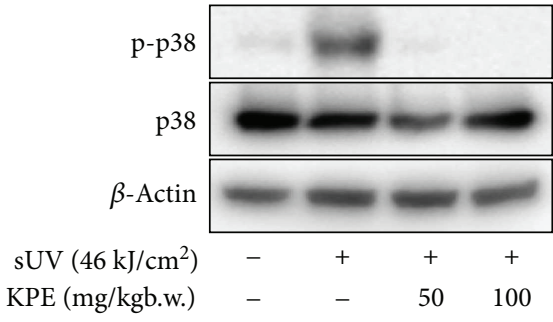

(b)

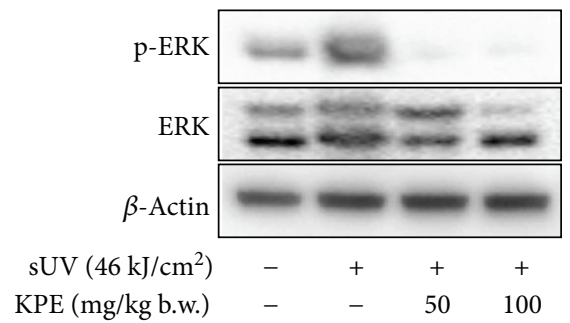

(c)

FIgURE 5: Effect of Kaempferia parviflora extract (KPE) on the sUV-induced MAPK signaling pathway in mouse skin. 50 and $100 \mathrm{mg} / \mathrm{kg}$ b.w. of KPE were treated to the abdominal skin of female SKH-1 hairless mice for $1 \mathrm{~h}$. Subsequently, $46 \mathrm{~kJ} / \mathrm{cm}^{2}$ of sUV was exposed to the mice. The protein level was quantified using Western blot analysis. Data are representative of 3 independent experiments that gave similar results. 


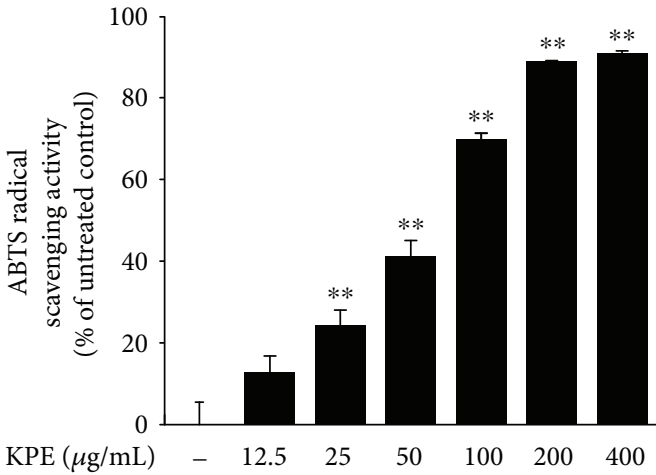

(a)

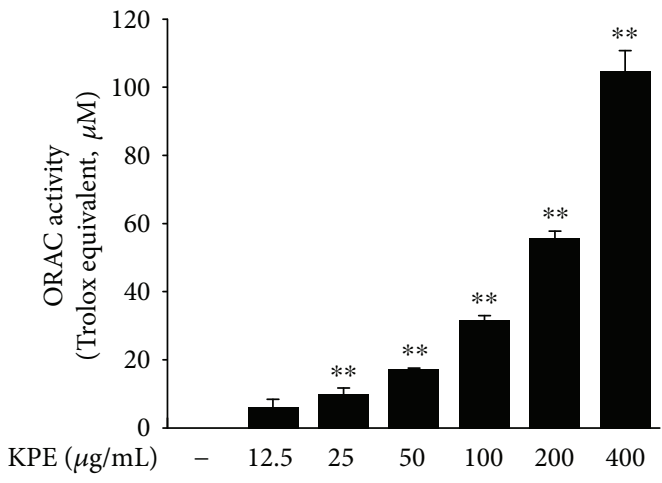

(c)

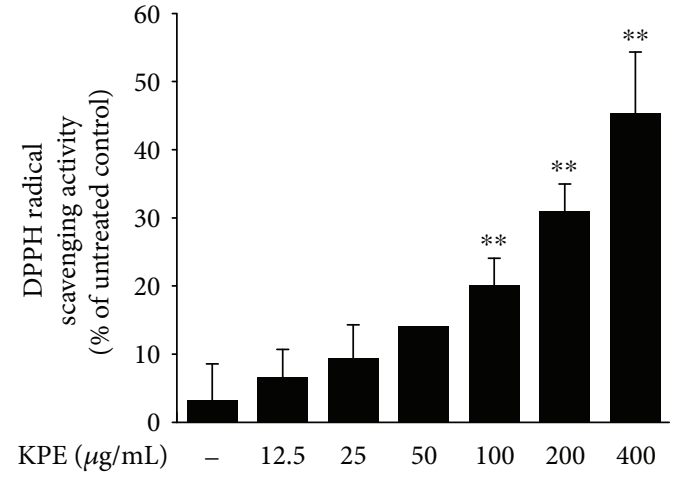

(b)

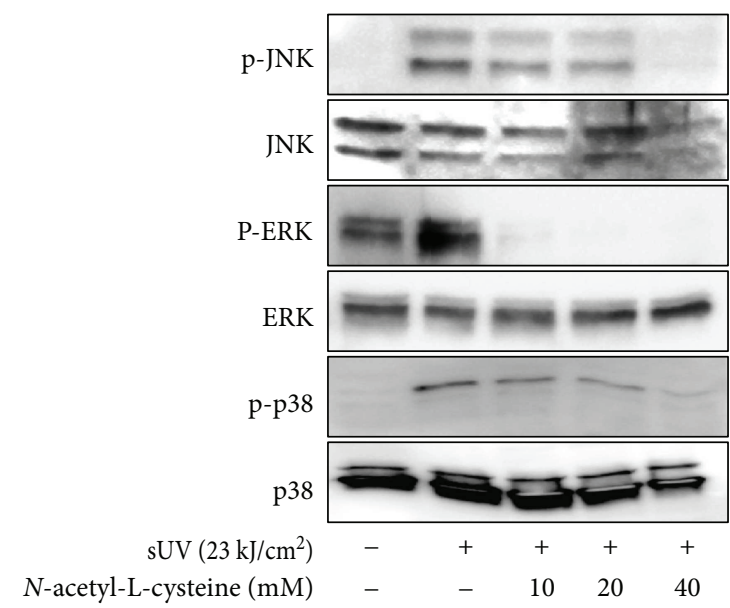

(e)

FIGURE 6: Antioxidative effect of Kaempferia parviflora extract (KPE) and the involvement of oxidative stress in sUV-induced inflammatory mechanisms. The antioxidative effect of KPE was measured using ABTS (a), DPPH (b), and ORAC (c) assay. (d) N-acetyl-L-cysteine (NAC) suppressed sUV-induced COX-2 expression (d) and MAPK phosphorylation (e). NAC was treated to the cells prior to $1 \mathrm{~h}$ of sUV irradiation. Each protein amount was measured using Western blot analysis with specific primary antibodies. Data are representative of 3 independent experiments that gave similar results. The asterisks $(* *)$ indicate the significant difference $($ of $p<0.001)$ compared to the untreated control.

3.4. Kaempferia parviflora Extract Inhibits sUV-Induced MKK4-JNK, MKK3/6-p38, and the MEK-ERK Signaling Pathway. The mitogen-activated protein kinase (MAPKs) family is a representative inflammatory signaling pathway that regulates $s U V$-induced COX-2 expression [8, 23]. MAPK kinases (MAP2Ks), MKK4, MKK3/6, and MEK directly activate MAPKs, JNK, p38, and ERK, respectively [24]. Thus, we confirmed the effect of KPE on sUV-induced MAPK activation. As seen in Figure 4, irradiation of sUV activated the MAPKK-MAPK signaling pathway, and pretreatment with KPE significantly alleviated MKK4-JNK, MKK3/6-p38, and MEK/ERK phosphorylation (Figure 4) in JB6 $\mathrm{P}^{+}$cells. To confirm these findings in vivo, 50 and $100 \mathrm{mg} / \mathrm{kg}$ b.w. treatments of KPE were applied to the dorsal skin of mice prior 
TABLE 1: Polyphenolic composition of KPE (unit: $\mu \mathrm{g} / \mathrm{mg}$ dry weight).

\begin{tabular}{lc}
\hline Analytes & Contents \\
\hline Ferulic acid & $1.80 \pm 0.40$ \\
Isorhamnetin & $0.61 \pm 0.13$ \\
Naringenin & $1.55 \pm 0.56$ \\
Luteolin & $1.00 \pm 0.23$ \\
Protocatechuic acid & $0.05 \pm 0.02$ \\
Caffeic acid & $0.86 \pm 0.15$ \\
Gentisic acid & $1.94 \pm 0.26$ \\
Hydroxybenzoic acid & $0.89 \pm 0.06$ \\
Gallic acid & $9.31 \pm 1.27$ \\
Apigenin & $2.37 \pm 0.14$ \\
Tectorigenin & $0.01 \pm 0.00$ \\
Malic acid & $0.57 \pm 0.05$ \\
Tangeretin & $2.15 \pm 0.19$ \\
\hline
\end{tabular}

All data are presented as mean \pm standard deviation $(n=3)$.

to sUV irradiation. It was observed that the phosphorylation of all MAPKs was downregulated in the presence of KPE treatment (Figure 5).

3.5. Kaempferia parviflora Extract Exhibits Antioxidative Activity. Excessive production of free radicals is harmful to the human body and enhances inflammatory signaling pathways $[25,26]$. Thus, suppressing agents against aberrant ROS production have been developed to attenuate inflammation [27-29]. Because KPE inhibited sUV-induced inflammatory signaling pathways, we sought to confirm the antioxidative activity using ABTS radicals. As seen in Figures 6(a) and 6(b), KPE exhibited dose-dependent ABTS and DPPH radical scavenging activity. Of particular note, almost $90 \%$ of the ABTS radicals were destroyed by $200 \mu \mathrm{g} / \mathrm{mL}$ of KPE treatment (Figure 6(a)). ORAC assay was also performed to verify the antioxidative activity of KPE. As seen in Figure 6(c), the dose-dependent Trolox equivalent antioxidant capacity was represented by KPE treatment. To evaluate the role of oxidative stress in $\mathrm{SUV}$-induced inflammation, we used $\mathrm{N}$-acetyl-L-cysteine (NAC), a well-known antioxidant chemical. Interestingly, we found a dramatic reduction of sUV-induced COX-2 expression by NAC treatment. Furthermore, the sUV-activated MAPK signaling pathway was significantly blocked by NAC treatment. Overall, we assumed that sUV exposure increases the inflammatory status by oxidative stress and KPE attenuated sUV-related inflammatory condition through antioxidative activity. Next, we analyzed the polyphenolic composition of KPE using UPLCMS/MS analysis (Supplementary Figure 1). Interestingly, KPE contained gallic acid $(9.31 \pm 1.27 \mu \mathrm{g} / \mathrm{mg}$ dry weight), apigenin $(2.37 \pm 0.14 \mu \mathrm{g} / \mathrm{mg}$ dry weight), and tangeretin $(2.15 \pm 0.19 \mu \mathrm{g} / \mathrm{mg}$ dry weight) (Table 1$)$.

\section{Discussion}

In the present study, an extract of Kaempferia parviflora (KPE) exhibited an attenuating effect on sUV-induced COX-2 expression in murine dorsal skin sections (Figure 1).
In addition, inflammatory MAPK signaling pathways were blocked in the presence of pretreatment with KPE. In 2009, Sae-wong et al. reported that KPE elicits anti-inflammatory activity via the suppression of iNOS and COX-2 mRNA expression [3]. We further confirmed this suppressive effect on COX-2 in vitro (Figure 2). KPE markedly inhibited sUV-induced COX-2 expression in keratinocytes sourced from human skin, suggesting that KPE may be useful in suppressing sunlight-mediated inflammation in human skin. The underlying mechanisms of the effect of KPE were shown to involve transcriptional regulation of COX-2 (Figure 3). Similar with our in vivo findings (Figure 5), the phosphorylation of various MAPKs was downregulated by KPE, as well as upstream regulators of the MAPKs. Notably, KPE demonstrated a potent dose-dependent radical scavenging effect (as seen in Figure 6(a)). UV stimulates reactive oxygen (ROS) generation and upregulates the expression of COX-2 and PGE2, which significantly contributes to skin damage and inflammation. In addition, UV-induced ROS activates MAPKs and inflammatory mediator enzymes as well as NF- $\kappa$ B and AP-1 $[30,31]$. Because aberrant ROS induction following sunlight exposure is a primary factor in sUVrelated skin inflammation, the antioxidative properties of $\mathrm{KPE}$ in a sUV-induced skin inflammation model were confirmed. Treatment with NAC, an ROS scavenger, inhibited the sUV-induced COX-2 expression as well as phosphorylation of c-Jun and MAPKs (Figures 6(d) and 6(e)). These results suggest that NAC and KPE have a similar effect by sUV-induced inflammatory responses, and it is associated with antioxidative activity. Collectively, we speculate that KPE may exert anti-inflammatory effects by regulating the intracellular level of oxidative stress.

To identify the bioactive compounds present in KPE, we analyzed its polyphenolic composition using UPLC-MS/MS. The primary compounds identified were gallic acid, apigenin, and tangeretin. The anti-inflammatory activities of gallic acid [32], apigenin [33, 34], and tangeretin [9] have previously been reported. Of particular note, Src kinase has been identified as a direct target of apigenin in a UVB-induced skin inflammation model [34]. Additionally, we have previously confirmed the anti-inflammatory effects of tangeretin in JB6 $\mathrm{P}^{+}$cells in 2011 [9]. Previously, several researches have tried to identify the bioactive compounds in $\operatorname{KPE}[35,36]$. Horigome et al. reported that 5,6-dimethoxyflavone (DMF) and 5-hydroxy-3,7,3', $4^{\prime}$-tetrymethoxyflavone (TMF) were found in KPE and these two compounds revealed that inhibition of the degranulation and the production and mRNA expression of inflammatory mediators [36].

Although further investigation is needed to more clearly understand the anti-inflammatory effects of KPE, our findings highlight the potential for KPE to be developed as an anti-inflammatory agent with various beneficial effects for skin health.

\section{Conflicts of Interest}

The authors declare that there are no competing interests associated with this publication. 


\section{Authors' Contributions}

Myung-hee Lee, Mi Jang, and Tae-Gyu Lim performed the experiments. Tae-Gyu Lim and Kyung-Tack Kim designed the project. Mi Jang, Hyo-Kyoung Choi, and Ah-Ram Han analyzed the results. Sung-Young Lee and Tae-Gyu Lim wrote the manuscript. All the authors have approved the final version of the manuscript.

\section{Acknowledgments}

This work was supported by the Korea Food Research Institute.

\section{Supplementary Materials}

The chemical composition of KPE. The analyses were performed using UPLC and mass spectrometry. The detailed procedure is presented in Materials and Methods. (Supplementary Materials)

\section{References}

[1] K. Sutthanut, B. Sripanidkulchai, C. Yenjai, and M. Jay, "Simultaneous identification and quantitation of 11 flavonoid constituents in Kaempferia parviflora by gas chromatography," Journal of Chromatography A, vol. 1143, no. 1-2, pp. 227-233, 2007.

[2] T. Azuma, Y. Tanaka, and H. Kikuzaki, "Phenolic glycosides from Kaempferia parviflora," Phytochemistry, vol. 69, no. 15, pp. 2743-2748, 2008.

[3] C. Sae-wong, P. Tansakul, and S. Tewtrakul, "Anti-inflammatory mechanism of Kaempferia parviflora in murine macrophage cells (RAW 264.7) and in experimental animals," Journal of Ethnopharmacology, vol. 124, no. 3, pp. 576-580, 2009.

[4] S. Tewtrakul, S. Subhadhirasakul, and S. Kummee, "Antiallergic activity of compounds from Kaempferia parviflora," Journal of Ethnopharmacology, vol. 116, no. 1, pp. 191-193, 2008.

[5] C. Rujjanawate, D. Kanjanapothi, D. Amornlerdpison, and S. Pojanagaroon, "Anti-gastric ulcer effect of Kaempferia parviflora," Journal of Ethnopharmacology, vol. 102, no. 1, pp. 120-122, 2005.

[6] T. Azuma, S.-i. Kayano, Y. Matsumura, Y. Konishi, Y. Tanaka, and H. Kikuzaki, "Antimutagenic and $\alpha$-glucosidase inhibitory effects of constituents from Kaempferia parviflora," Food Chemistry, vol. 125, no. 2, pp. 471-475, 2011.

[7] F. Ali, B. A. Khan, and S. Sultana, "Wedelolactone mitigates UVB induced oxidative stress, inflammation and early tumor promotion events in murine skin: plausible role of NFkB pathway," European Journal of Pharmacology, vol. 786, pp. 253264, 2016.

[8] T.-G. Lim, J.-E. Kim, S. K. Jung et al., "MLK3 is a direct target of biochanin A, which plays a role in solar UV-induced COX-2 expression in human keratinocytes," Biochemical Pharmacology, vol. 86, no. 7, pp. 896-903, 2013.

[9] J. H. Yoon, T. G. Lim, K. M. Lee, A. J. Jeon, S. Y. Kim, and K. W. Lee, "Tangeretin reduces ultraviolet B (UVB)-induced cyclooxygenase-2 expression in mouse epidermal cells by blocking mitogen-activated protein kinase (MAPK) activation and reactive oxygen species (ROS) generation," Journal of Agricultural and Food Chemistry, vol. 59, no. 1, pp. 222-228, 2011.

[10] S. K. Jung, S. J. Ha, Y. A. Kim et al., "MLK3 is a novel target of dehydroglyasperin $\mathrm{D}$ for the reduction in UVB-induced COX2 expression in vitro and in vivo," Journal of Cellular and Molecular Medicine, vol. 19, no. 1, pp. 135-142, 2015.

[11] S.-C. Tang, P.-Y. Liao, S.-J. Hung et al., “Topical application of glycolic acid suppresses the UVB induced IL-6, IL-8, MCP-1 and COX-2 inflammation by modulating NF- $\kappa \mathrm{B}$ signaling pathway in keratinocytes and mice skin," Journal of Dermatological Science, vol. 86, no. 3, pp. 238-248, 2017.

[12] L. Y. Pang, E. A. Hurst, and D. J. Argyle, "Cyclooxygenase-2: a role in cancer stem cell survival and repopulation of cancer cells during therapy," Stem Cells International, vol. 2016, Article ID 2048731, 11 pages, 2016.

[13] S. Grösch, E. Niederberger, and G. Geisslinger, "Investigational drugs targeting the prostaglandin E2 signaling pathway for the treatment of inflammatory pain," Expert Opinion on Investigational Drugs, vol. 26, no. 1, pp. 51-61, 2017.

[14] C. J. Smith, Y. Zhang, C. M. Koboldt et al., "Pharmacological analysis of cyclooxygenase-1 in inflammation," Proceedings of the National Academy of Sciences of the United States of America, vol. 95, no. 22, pp. 13313-13318, 1998.

[15] M. A. Iniguez, A. Rodriguez, O. V. Volpert, M. Fresno, and J. M. Redondo, "Cyclooxygenase-2: a therapeutic target in angiogenesis," Trends in Molecular Medicine, vol. 9, no. 2, pp. 73-78, 2003.

[16] S. Byun, K. W. Lee, S. K. Jung et al., "Luteolin inhibits protein kinase $\mathrm{C} \varepsilon$ and c-Src activities and UVB-induced skin cancer," Cancer Research, vol. 70, no. 6, pp. 2415-2423, 2010.

[17] R. van den Berg, G. R. M. M. Haenen, H. van den Berg, and A. Bast, "Applicability of an improved Trolox equivalent antioxidant capacity (TEAC) assay for evaluation of antioxidant capacity measurements of mixtures," Food Chemistry, vol. 66, no. 4, pp. 511-517, 1999.

[18] M. Wang, Y. Jin, and C.-T. Ho, "Evaluation of resveratrol derivatives as potential antioxidants and identification of a reaction product of resveratrol and 2,2-diphenyl-1-picryhydrazyl radical," Journal of Agricultural and Food Chemistry, vol. 47, no. 10, pp. 3974-3977, 1999.

[19] M. Grewe, U. Trefzer, A. Ballhorn, K. Gyufko, H. Henninger, and J. Krutmann, "Analysis of the mechanism of ultraviolet (UV) B radiation-induced prostaglandin E2 synthesis by human epidermoid carcinoma cells," Journal of Investigative Dermatology, vol. 101, no. 4, pp. 528-531, 1993.

[20] A. von Knethen, D. Callsen, and B. Brüne, "Superoxide attenuates macrophage apoptosis by NF- $\kappa$ B and AP-1 activation that promotes cyclooxygenase-2 expression," Journal of Immunology, vol. 163, no. 5, pp. 2858-2866, 1999.

[21] J.-E. Kim and K. Lee, "Silkworm thorn stem extract targets RSK2 and suppresses solar UV-induced cyclooxygenase-2 expression," International Journal of Molecular Sciences, vol. 16, no. 12, pp. 25096-25107, 2015.

[22] S. Y. Lee, J. Yoon, M. H. Lee et al., "The role of heterodimeric AP-1 protein comprised of JunD and c-Fos proteins in hematopoiesis," Journal of Biological Chemistry, vol. 287, no. 37, pp. 31342-31348, 2012.

[23] K. C. N. Chang, Y. Wang, I. G. Oh et al., "Estrogen receptor $\beta$ is a novel therapeutic target for photoaging," Molecular Pharmacology, vol. 77, no. 5, pp. 744-750, 2010. 
[24] A. M. Winter-Vann and G. L. Johnson, "Integrated activation of MAP3Ks balances cell fate in response to stress," Journal of Cellular Biochemistry, vol. 102, no. 4, pp. 848-858, 2007.

[25] R. Loperena and D. G. Harrison, "Oxidative stress and hypertensive diseases," Medical Clinics of North America, vol. 101, no. 1, pp. 169-193, 2017.

[26] S. I. Liochev, "Reactive oxygen species and the free radical theory of aging," Free Radical Biology and Medicine, vol. 60, pp. 1-4, 2013.

[27] M. Reinisalo, A. Kårlund, A. Koskela, K. Kaarniranta, and R. O. Karjalainen, "Polyphenol stilbenes: molecular mechanisms of defence against oxidative stress and aging-related diseases," Oxidative Medicine and Cellular Longevity, vol. 2015, Article ID 340520, 24 pages, 2015.

[28] P. Poprac, K. Jomova, M. Simunkova, V. Kollar, C. J. Rhodes, and M. Valko, "Targeting free radicals in oxidative stressrelated human diseases," Trends in Pharmacological Sciences, vol. 38, no. 7, pp. 592-607, 2017.

[29] R. Jiao, Y. Liu, H. Gao, J. Xiao, and K. F. So, “The anti-oxidant and antitumor properties of plant polysaccharides," The American Journal of Chinese Medicine, vol. 44, no. 03, pp. 463-488, 2016.

[30] A. Svobodova, D. Walterova, and J. Vostalova, "Ultraviolet light induced alteration to the skin," Biomedical Papers, vol. 150, no. 1, pp. 25-38, 2006.

[31] S. Y. Buckman, A. Gresham, P. Hale et al., "COX-2 expression is induced by UVB exposure in human skin: implications for the development of skin cancer," Carcinogenesis, vol. 19, no. 5, pp. 723-729, 1998.

[32] M. Tsang, D. Jiao, B. Chan et al., “Anti-inflammatory activities of pentaherbs formula, berberine, gallic acid and chlorogenic acid in atopic dermatitis-like skin inflammation," Molecules, vol. 21, no. 12, p. 519, 2016.

[33] M. Q. Man, M. Hupe, R. Sun, G. Man, T. M. Mauro, and P. M. Elias, "Topical apigenin alleviates cutaneous inflammation in murine models," Evidence-based Complementary and Alternative Medicine, vol. 2012, Article ID 912028, 7 pages, 2012.

[34] S. Byun, J. Park, E. Lee et al., "Src kinase is a direct target of apigenin against UVB-induced skin inflammation," Carcinogenesis, vol. 34, no. 2, pp. 397-405, 2013.

[35] K. Ninomiya, T. Matsumoto, S. Chaipech et al., "Simultaneous quantitative analysis of 12 methoxyflavones with melanogenesis inhibitory activity from the rhizomes of Kaempferia parviflora," Journal of Natural Medicines, vol. 70, no. 2, pp. 179-189, 2016.

[36] S. Horigome, I. Yoshida, A. Tsuda et al., "Identification and evaluation of anti-inflammatory compounds from Kaempferia parviflora," Bioscience, Biotechnology, and Biochemistry, vol. 78, no. 5, pp. 851-860, 2014. 


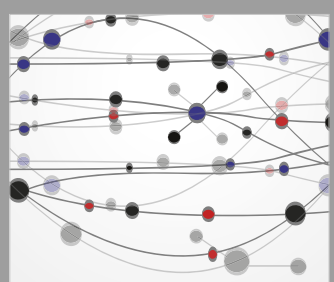

The Scientific World Journal
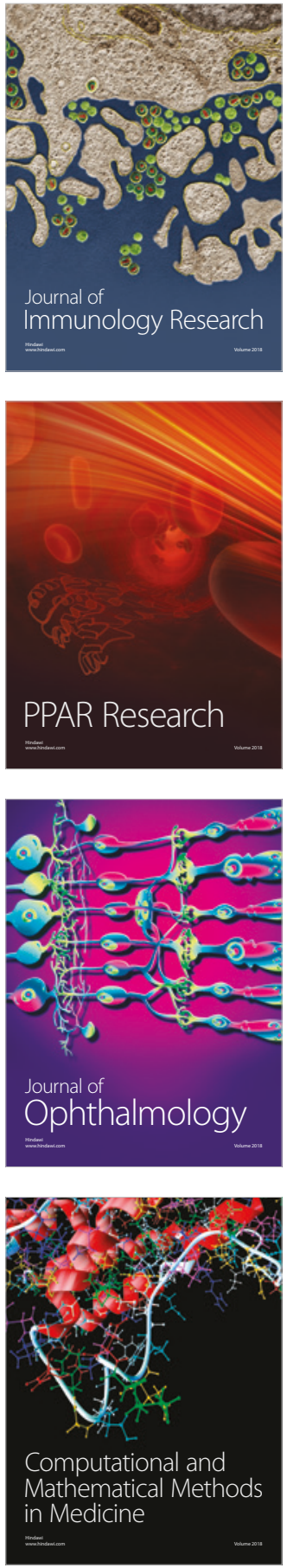

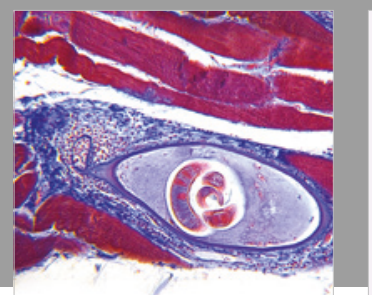

Gastroenterology Research and Practice

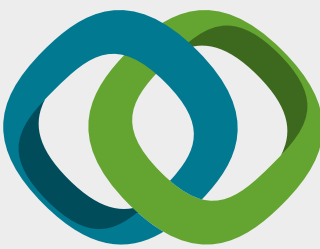

\section{Hindawi}

Submit your manuscripts at

www.hindawi.com
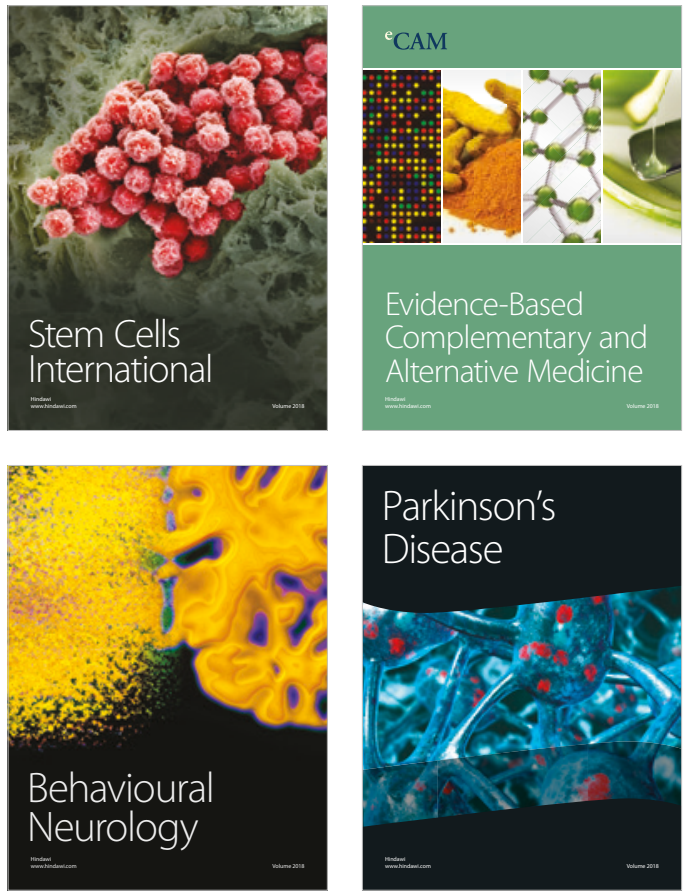

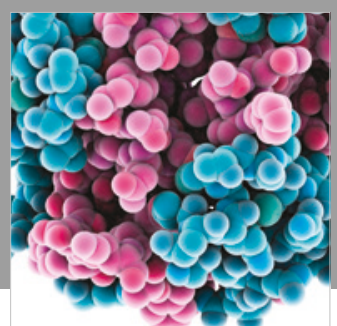

ournal of

Diabetes Research

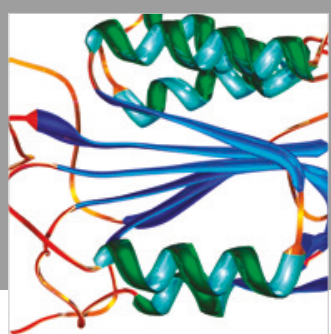

Disease Markers
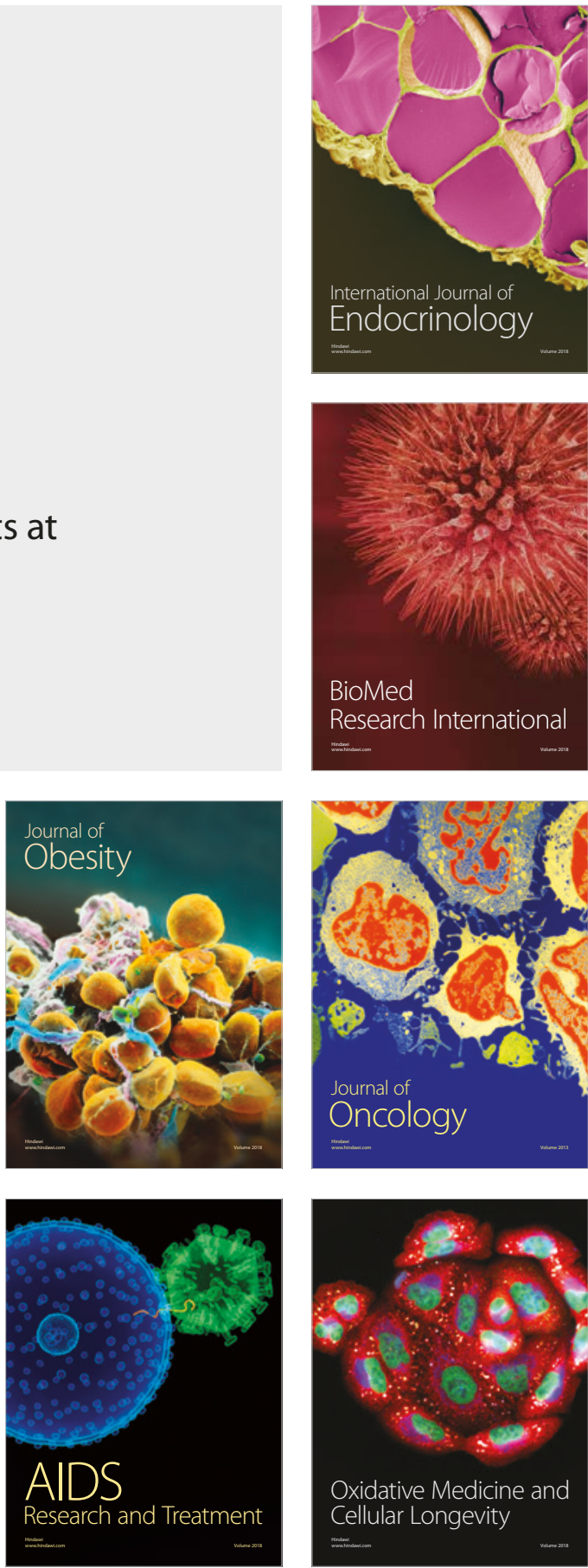